\title{
Attachment-based family therapy for adolescents with suicidal ideation: a randomized controlled trial.
}

\author{
Guy S. Diamond \\ The Children's Hospital of Philadelphia, University of Pennsylvania School of Medicine \\ Matthew B. Wintersteen \\ Thomas Jefferson University \\ Gregory K. Brown \\ University of Pennsylvania School of Medicine
}

Gary M. Diamond

Ben-Gurion University of the Negev, Israel

Eollow this and additional works at: https://jdc.jefferson.edu/phbfp Robert Gallop

stachestevelvividersity and Health Commons, and the Psychiatry Commons

Let us know how access to this document benefits you

See next page for additional authors

Recommended Citation

Diamond, Guy S.; Wintersteen, Matthew B.; Brown, Gregory K.; Diamond, Gary M.; Gallop, Robert;

Shelef, Karni; and Levy, Suzanne, "Attachment-based family therapy for adolescents with suicidal ideation: a randomized controlled trial." (2010). Department of Psychiatry and Human Behavior Faculty Papers. Paper 8.

https://jdc.jefferson.edu/phbfp/8

This Article is brought to you for free and open access by the Jefferson Digital Commons. The Jefferson Digital Commons is a service of Thomas Jefferson University's Center for Teaching and Learning (CTL). The Commons is a showcase for Jefferson books and journals, peer-reviewed scholarly publications, unique historical collections from the University archives, and teaching tools. The Jefferson Digital Commons allows researchers and interested readers anywhere in the world to learn about and keep up to date with Jefferson scholarship. This article has been accepted for inclusion in Department of Psychiatry and Human Behavior Faculty Papers by an authorized administrator of the Jefferson Digital Commons. For more information, please contact: JeffersonDigitalCommons@jefferson.edu. 


\section{Authors}

Guy S. Diamond, Matthew B. Wintersteen, Gregory K. Brown, Gary M. Diamond, Robert Gallop, Karni Shelef, and Suzanne Levy 


\title{
As submitted to:
}

Journal of the American Academy of Child and Adolescent Psychiatry

\author{
And later published as: \\ Volume 49. Issue 2. Pages 122-131 (February 2010) \\ “Attachment-Based Family Therapy for Adolescents with \\ Suicidal Ideation:
}

A Randomized Controlled Trial"

Guy S. Diamond, Ph.D.

The Children's Hospital of Philadelphia

University of Pennsylvania School of Medicine

Matthew B. Wintersteen, Ph.D.

Thomas Jefferson University

Gregory K. Brown, Ph.D.

University of Pennsylvania School of Medicine

Gary M. Diamond, Ph.D.

Ben-Gurion University of the Negev, Israel

Robert Gallop, Ph.D.

West Chester University

Karni Shelef, Ph.D.

Achva Academic College, Israel

Suzanne Levy, Ph.D.

The Children's Hospital of Philadelphia

Guy S. Diamond, Ph.D.

Center for Family Intervention Science

Children's Hospital of Philadelphia

34th and Civic Center Blvd.

Philadelphia, PA 19104

215-590-7550 (Phone)

215-590-7410 (fax) 
diamondg@email.chop.edu

This study was funded by grant R49 CE000428 from the Centers for Disease Control and Prevention (Dr. G.S. Diamond, PI).

Trial Registration: Clinicaltrials.gov identifier: NCT00604097;

http://clinicaltrials.gov/ct2/show/NCT00604097?term=NCT00604097\&rank=1

Word Count: 5,995 words

Published February 2010 in the Journal of the American Academy of Child and Adolescent Psychiatry (www.jaacap.com). 
Family Therapy for Suicidal Youth

\begin{abstract}
Objective: To evaluate whether Attachment-Based Family Therapy (ABFT) is more effective than Enhanced Usual Care (EUC) for reducing suicidal ideation and depressive symptoms in adolescents. Method: Randomized controlled trial of suicidal adolescents between the ages of 12 and 17, identified in primary care and emergency departments. Of 341 adolescents screened, 66 (70\% African American) entered the study for 3 months of treatment. Assessment occurred at baseline, six weeks, 12 weeks, and 24 weeks. ABFT consisted of individual and family meetings, and EUC consisted of a facilitated referral to other providers. All participants received weekly monitoring and access to a 24-hour crisis phone. Trajectory of change and clinical recovery were measured for suicidal ideation and depressive symptoms. Results: Using intent-to-treat, patients in ABFT demonstrated significantly greater rates of change on selfreported suicidal ideation at post-treatment and benefits were maintained at follow-up, with a strong overall effect size $(\mathrm{ES}=.97)$. Between-group differences were similar on clinician ratings. Significantly more patients in ABFT met criteria for clinical recovery on suicidal ideation posttreatment (87\%; 95\% CI, 74.6-99.6) than patients in EUC (51.7\%; 95\% CI, 32.4-54.32). Benefits were maintained at follow-up (ABFT, 70\%; 95\% CI, 52.6-87.4; EUC 34.6\%; 95\% CI, 15.654.2), with a strong effect size $(\mathrm{OR}=4.41)$. Patterns of depressive symptoms over time were similar, as were results for a subsample of adolescents with diagnosed depression. Conclusions: ABFT is more efficacious than EUC in reducing suicidal ideation and depressive symptoms in adolescents. Additional research is warranted to confirm treatment efficacy and test the proposed mechanism of change.
\end{abstract}

Keywords: adolescents, suicide, family therapy 
Family Therapy for Suicidal Youth

\section{Introduction}

Suicide is the third leading cause of death in American adolescents, accounting for 1,371 deaths of youth between the ages of 12 and 18 in $2005 .{ }^{1}$ In addition, every year nearly one million adolescents attempt suicide, leading to high emotional costs for families and financial costs for the health system. ${ }^{2}$ Unfortunately, no medication and few (less than 10) psychotherapy studies have focused on suicidal youth. ${ }^{3}$ Existing studies demonstrate only a slight reduction in suicide attempts or ideation, and experimental treatments have rarely been superior to treatment as usual. ${ }^{4-7}$ Cognitive Behavioral Therapy $(\mathrm{CBT})^{8}$ and/or medication successfully reduced mild suicidal ideation in a depressed sample (TADS 12), but not in a more severely depressed and suicidal population.$^{9}$ Overall, the efficacy of CBT and other forms of psychotherapy for suicidal youth has been mixed, ${ }^{3,10}$ and debate continues over whether medication exacerbates or reduces suicidal thinking. ${ }^{11}$ Clearly, research on alternative intervention approaches is warranted.

Family-based treatments for youth suicide are promising for a number of reasons. First, negative family functioning (e.g., high conflict, low cohesion, poor attachment, ineffective parenting, etc.) is a strong risk factor for youth suicide and depression. ${ }^{12}$ Family conflict may precede $20 \%$ of suicide deaths and $50 \%$ of non-fatal suicidal acts. ${ }^{13}$ Second, family conflict is frequently a negative moderator of treatment outcome. ${ }^{8,9,14}$ Third, family cohesion, emotional support, and appropriate supervision are protective factors in preventing suicide and depression. $^{12}$

Not surprisingly, several family intervention studies have shown promise for treating suicidal youth. Harrington et al., found routine care plus family intervention more effective at reducing suicidal ideation than routine care alone, but only for youth without major depression. ${ }^{15}$ Huey et al. found Multisystemic Family Therapy more effective than hospitalization for 
decreasing suicide attempts but not suicidal ideation, depressive affect, or hopelessness. ${ }^{16}$ Brent et al., found CBT and family therapy for depressed adolescents had significant reductions in suicidal ideation compared to supportive therapy post-treatment, but no difference at follow-up. ${ }^{14}$

One promising intervention is ABFT, the first manualized family therapy specifically designed to target family processes associated with depression and suicide. ABFT aims to improve the family's capacity for problem solving, affect regulation, and organization. This strengthens family cohesion, which can buffer against suicidal thinking, depression, and risk behaviors. ${ }^{12}$ In a study of ABFT for depressed adolescents, the treatment was found to significantly reduce suicidal ideation. ${ }^{17}$

This study tested the efficacy of ABFT for reducing suicidal ideation in adolescents. This study was designed and powered specifically to examine reductions in suicidal ideation. While many adolescents may think about suicide sometimes, severe and persistent ideation is a serious risk factor for suicidal behavior. ${ }^{2,3,18}$ In addition, these youth present a worrisome challenge for parents, teachers, mental health and medical professionals, all of whom fear that ideation may lead to attempts. Unfortunately, ideators are often excluded from clinical trials because of the safety risks ${ }^{19}$ and no prevention or treatment studies target youth with severe ideation. Moreover, few studies have included a large sample of inner city, minority youth, a group whose risk for suicide has risen dramatically in the last decade. ${ }^{20}$ With funding from the Centers for Disease Control and Prevention, we aimed to test whether ABFT reduced suicidal ideation and depressive symptoms more than Enhanced Usual Care (EUC). Because treatment retention has been a challenge in treating suicidal youth, ${ }^{21,22}$ we also aimed to see if ABFT had better retention than treatment in the community. 
Family Therapy for Suicidal Youth

\section{Method}

\section{Procedures}

The research occurred in the Department of Psychiatry at the Children's Hospital of Philadelphia (CHOP). Patients were recruited mainly from CHOP's primary care offices and emergency room. During routine clinical interviews, patients identified as having suicidal thoughts were referred to the clinic social worker for an assessment. Adolescents who endorsed scores above 31 on the Suicidal Ideation Questionnaire (SIQ-JR) ${ }^{23}$ and above 20 on the Beck Depression Inventory (BDI-II) ${ }^{24}$ (i.e., moderate depression) were referred to our study. On a second screening, generally two days later $(M=2.21$ days, $\mathrm{SD}=1.34)$, if both suicide and depression scores remained above the cut-offs, patients were eligible for the study. For this highrisk group, a longer waiting period was deemed unethical. Patients had to be between 12 and 17 years old with a parent or guardian willing to participate.

Adolescents were not eligible if they 1) needed psychiatric hospitalization, 2) were recently discharged from a psychiatric hospital, 3) had current psychosis, or 4) had mental retardation or history of borderline intellectual functioning. Patients could remain on antidepressant medication if they had started it at least 12 weeks prior to randomization.

Eligible adolescents and parents signed a written, informed consent and were then randomized to either ABFT or EUC. Assessments were conducted at baseline, six weeks, 12 weeks (post-treatment), and 24 weeks (follow-up, 12 weeks after treatment). All assessment staff had a B.A. in psychology and were trained, certified and supervised by the project coordinator. The study was approved by the IRB at CHOP.

We used an adaptive or "urn" randomization procedure, maintained by the statistician, with four stratification variables: age, gender, past suicide attempt, and family conflict. The 
project coordinator was blind to the randomization sequence until the baseline assessment was complete. Given limited grant resources and clinical severity of the patients, blinding assessors to treatment assignment was not feasible.

Seven Ph.D. or MSW-level therapists provided ABFT (4 female, 3 male). Six were Caucasian and one was Asian-American. Each were trained, certified, and supervised by the first and fourth authors. Fidelity to treatment was monitored through weekly case discussion, videotape review, and live supervision.

\section{Treatments}

Attachment-Based Family Therapy (ABFT). ABFT ${ }^{17,25}$ emerges from interpersonal theories that suggest depression and suicide can be precipitated, exacerbated, or buffered against by the quality of family relationships. ${ }^{26}$ Therefore, ABFT focuses on strengthening parentadolescent attachment bonds to create a protective and secure base for adolescent development. Therapy begins with a discussion of what prohibits the adolescent from turning to his or her parent(s) for help when contemplating suicide. Family barriers ranged from a) stress due to the adolescent's depression, b) a history of negative interactions and communication, c) abuse, neglect, abandonment and or d) parental psychopathology. Even well-functioning families needed help effectively managing these suicidal crises.

Although ABFT therapists implement behavior focused and psychoeducational interventions, the model is primarily a process oriented, emotion focused treatment, guided by a semi-structured treatment protocol. Treatment is conceptualized as five specific tasks, each with a distinct process and goal. Treatment begins with the Relational Reframe Task, conducted with relevant family members, and aims to strengthen and or repair family relationships (as opposed to behavior management) as the primary initial treatment goal. Then the Adolescent Alliance 
Task, with the adolescent alone, identifies core family conflicts linked to depression and prepares the adolescent to discuss these with his or her parents (1 to 2 sessions).

Next, the Parent Alliance Task with the parents alone aims to amplify parental love and empathy, and to teach emotionally focused parenting skills that will increase affective attunement ( 1 to 2 sessions). These sessions culminate in the Reattachment Task, where families come back together to discuss identified problems and practice new communication, problem solving, and affect regulation skills (3 to 4 sessions). As attachment relationships strengthen, therapists focus on the Competency Task. This task promotes adolescent autonomy (e.g., improving school functioning, increasing pleasant activities, etc.) while maintaining family connection. This ideal progression of tasks is modified as needed.

Enhanced Usual Care (EUC). EUC was a facilitated referral process with ongoing clinical monitoring. We found providers, set up initial appointments, and encouraged attendance. It was the treating therapist's responsibility to engage and retain the adolescent in treatment.

Safety Monitoring. We monitored patient's clinical status weekly using the SIQ-JR and BDI-II, administered either face-to-face (ABFT) or over the phone (EUC). Information regarding service utilization was gathered weekly. In addition, project staff were on call all the time in case of emergency.

\section{Outcome Measures}

All outcome assessments were conducted face to face for all patients. The study had two declared primary outcomes: suicidal ideation and depressive symptoms. The self-report SIQ-JR ${ }^{23}$ and assessor-administered Scale for Suicidal Ideation $(\mathrm{SSI})^{27}$ were used to assess trajectory of change in suicidal ideation over time (total scores). For clinical recovery, we used the SIQ-JR $<13$ (based on a non clinical inner city, minority sample ${ }^{28}$ and SSI $=0$ ). The SSI was 
dichotomized because the data was not normally distributed, and to evaluate the treatment based on the absence of ideation. ${ }^{29}$ The self-report BDI-II was used to assess trajectory of change (total score) in depressive symptoms and clinical response (BDI-II $\leq 9) .{ }^{14,24}$

Treatment retention was a secondary outcome, measured as number of sessions attended. For descriptive purposes and subgroup analyses, psychiatric diagnosis was determined using the clinician-administered, computerized Diagnostic Interview Schedule for Children (DISC). ${ }^{30}$

\section{Data Analysis}

\section{Statistical Methods}

Tests of baseline differences in demographic and clinical characteristics were conducted using independent samples t-tests for continuous variables and Chi-square or Fisher exact tests of independence for categorical variables. Implementation of various Box-Cox transformations indicated a square-root transformation would correct the deviations in normality for the SIQ-JR and the BDI-II (both were negatively skewed). No substantial improvement in near normality was achieved for the SSI, so it was dichotomized. ${ }^{29}$

The outcome analyses examined change across all four assessments. Hierarchical linear modeling (HLM) was used to investigate treatment differences using the intent-to-treat sample. ${ }^{31}$ For the SIQ-JR and the SSI, the main efficacy analysis was determined to be piecewise, modeling the rate of change from baseline to 12 weeks (end of active treatment), and then again at 24 weeks (follow-up). For the BDI-II, the piecewise model ran first from baseline to 6 weeks (mid-treatment) and then to 24 weeks. For the binary outcomes, including clinical response, we implemented a hierarchical generalized linear model (HGLM), which accommodates the binary nature of the data, as well as the clustering within each subject. Effect size was measured using Cohen's d for the continuous measures and odds ratios (OR) for the binary measures. The OR 
corresponded to, on average, the number of times an event was more likely to occur for ABFT compared to EUC at each assessment.

Nonparametric Wilcoxon Rank sum tests were used to compare number of sessions attended. Chi-square analyses were used to assess treatment retention at each assessment. The pattern-mixture model approach was used to assess whether missing data had a substantive influence on results. All analyses were conducted using SPSS Version 15 and SAS Version 9.1.3.

\section{Statistical Power}

We originally estimated a required sample size of 120 patients for the longitudinal analysis of SIQ-JR. Given the time needed for creating a referral infrastructure with primary care and the short recruitment window (26 months), we did not achieve the targeted goals. The obtained sample sizes of 35 (ABFT) and 31 (EUC), with a 14.4\% missing data rate at the 24 week follow-up assessment, and an approximate intra-subject correlation of .40 (estimated value of 0.34 for the SIQ-JR scale), indicated the study power of $74.5 \%$. Such power would yield a statistically significant result for an effect size of 0.60 , power of $81.0 \%$ for an effect size of 0.65 , and power of $86.4 \%$ for an effect size of 0.70 .

\section{Results}

\section{Enrollment Statistics}

Between March 2005 and May 2007, 341 adolescents were referred for the first screening (Figure 1). Of the 248 who were excluded, 188 did not meet study criteria, 36 became unreachable prior to the second screening, 14 refused study participation, six were hospitalized, and four were excluded for other reasons. Of the 93 who attended the second screening, 11 no longer met study criteria, eight were unreachable prior to consent, and five refused study 
Family Therapy for Suicidal Youth

participation. Of the 69 who qualified based on the second screening, three reported psychotic features during intake and were excluded. Of the final sample, 50 participants (75\%) were referred from primary care, and $16(25 \%)$ were referred from emergency departments.

\section{Demographic, Clinical, and EUC Characteristics}

There were no differences on any demographic or clinical variables between the two treatment conditions (Table 1$)$. The mean age was 15.1 years $(\mathrm{SD}=1.5), 49(74 \%)$ were African American, and $55(83 \%)$ were female. Of the parents, 27 (41\%) had an income under $\$ 30,000$, $17(26 \%)$ were married and $46(70 \%)$ had no more than a high school diploma. Thirty-one (47\%) adolescents met criteria for a depressive disorder, 44 (67\%) met criteria for any anxiety disorder, and $38(57 \%)$ met criteria for an externalizing disorder (i.e., ADHD, oppositional, or conduct disorder). Forty-one $(62 \%)$ participants reported having made a past suicide attempt, and nearly three quarters of those patients (30 of the 41 attempters) reported making multiple attempts. Twenty (30\%) participants reported a history of suicide attempts in their families. Upon study entry, six patients were stable (more than 12 weeks) on antidepressant medication, three in each treatment condition.

Of the patients in EUC, seven (23\%) were referred to private practice, and 24 (77\%) were referred to community mental health centers. Sixteen $(52 \%)$ received individual therapy, six (19\%) received group therapy, two $(6 \%)$ received family therapy, one $(3 \%)$ received case management, and six (19\%) did not attend treatment. There were no differences in the completion rates of assessments between the two treatment groups at any of the time points. Implementation of the pattern-mixture approach indicated group comparisons were not biased due to completion status. 
Family Therapy for Suicidal Youth

\section{Trajectory of Change: Suicidal Ideation}

On self-reported suicidal ideation (i.e., SIQ-JR), ABFT demonstrated a significantly greater rate of improvement during the treatment period $(12$ weeks) than $\operatorname{EUC}(F(1,64)=12.60$, $P=0.001$ ) (Figure 2; Table 2). No significant difference in rates of change was observed between treatments during the follow-up phase $(F(1,64)=0.01, P<0.91)$. During the treatment phase, the amount of change on the square-root of the SIQ-JR scale was -5.32 $(s e=0.38)$ for $\mathrm{ABFT}$ and $-3.35(s e=0.40)$ for EUC. This corresponded to a significant difference of $-1.97(s e=$ $0.55)$ with a large effect size $(d=.95)$ in favor of ABFT $(t(64)=3.55, P=0.0007)$. The total change from baseline to follow-up on the square-root of the SIQ-JR for ABFT was -4.37 ( $s e=$ $0.40)$ and for EUC -2.34 (se = 0.43). This corresponded to a significant difference of 2.03 (se= $0.59)$ with a large effect size $(d=0.97)$, in favor of ABFT $(t(64)=-3.45, P=0.001)$. Therefore, despite the slight increase of suicidal ideation for both groups during the follow-up phase, the large effect in favor of ABFT was maintained.

Regarding the trajectory of change of clinician-rated suicidality (SSI), ABFT demonstrated a significantly greater rate of change on the log-odds scale than EUC during the treatment phase $(F(1,64)=6.32, P=0.014)$. During the follow-up phase, there was no significant difference in rate of change between the two groups $(F(1,64)=0.20, P=0.65)$. During the treatment phase, the amount of change on the log-odds SSI for ABFT was $2.51(s e=0.51)$ and for EUC was 0.77 (se $=0.47)$. This corresponded to a significant difference of $1.74(s e=0.69)$ with a medium to larger effect size $(d=0.62)$ in favor of ABFT $(t(64)=2.51, P=0.014)$. The total change from baseline to follow-up on the log-odds SSI scale for ABFT was $3.21(s e=0.61)$ and for EUC $1.14(s e=0.53)$. This corresponded to a significant difference of $2.07(s e=0.80)$ with a medium to large effect size $(d=0.64)$ in favor of ABFT $(t(64)=2.58, P=0.012)$. 
Family Therapy for Suicidal Youth

Therefore, while both groups improved after 12 weeks, ABFT continued to have a slightly higher rate of improvement, with a medium to large effect.

\section{Clinical Recovery: Suicidal Ideation}

For clinically meaningful change on the self-reported SIQ-JR (Table 3), ABFT had a significant effect at all time points and large effect sizes represented by the odds ratios. At six weeks, $69.7 \%$ of ABFT participants and $40.7 \%$ of EUC participants reported suicidal ideation in the normative range $\left(\mathrm{OR}=3.35,95 \% \mathrm{CI}: 1.15-9.73 ; \chi^{2}(1)=5.07, P=0.02\right)$. At 12 weeks, $87.1 \%$ of ABFT participants and $51.7 \%$ of EUC participants reported ideation in the normative range $\left(\mathrm{OR}=6.30,95 \% \mathrm{CI}: 1.76-22.61, \chi^{2}(1)=8.93, P=0.003\right)$. At 24 weeks, $70.0 \%$ of ABFT participants and $34.6 \%$ of EUC participants reported ideation in the normative range $(\mathrm{OR}=4.41$, 95\% CI: $\left.1.43-13.56 ; \chi^{2}(1)=7.01, P=0.008\right)$.

Clinical response on clinician-rated SSI demonstrated similar patterns as the SIQ-JR. At six weeks, $40.7 \%$ of ABFT participants and $33.3 \%$ of EUC participants reported no suicidal ideation in the past week $\left(\mathrm{OR}=1.38,95 \% \mathrm{CI}: 0.44-4.32 ; \chi^{2}(1)=0.30, P=0.59\right)$. At 12 weeks, $69.2 \%$ of the ABFT participants and $34.6 \%$ of the EUC participants reported no suicidal ideation in the past week $\left(\mathrm{OR}=4.25,95 \% \mathrm{CI}: 1.33-13.56 ; \chi^{2}(1)=6.24, P=0.01\right)$. At 24 weeks, $82.1 \%$ of the ABFT participants and $46.2 \%$ of the EUC participants reported no suicidal ideation in the past week $\left(\mathrm{OR}=5.37,95 \% \mathrm{CI}: 1.56-18.49 ; \chi^{2}(1)=7.66, P=0.006\right)$. Over the six months of the study, 11 patients made low lethality suicide attempts $(\mathrm{ABFT}=4$; EUC $=7)$. These numbers are too small for statistical analysis.

\section{Depressive Symptoms}

Trajectory of change on self-reported depressive symptoms (BDI-II) showed a marginally significant differential rate of change during the first six weeks of treatment between the two 
Family Therapy for Suicidal Youth

groups $(F(1,64)=3.00, P=0.09)$. From mid-treatment to follow-up, there was no significant difference in rate of change between the two groups $(F(1,64)=0.33, P=0.57)$. During the first six weeks, ABFT demonstrated an average reduction of $2.16(s e=0.24)$ while EUC had an average reduction of $1.55(s e=0.26)$ on the square root BDI-II. This corresponded to a near significant difference of $0.62(s e=0.353)$ with a small to medium effect size $(d=0.37 ; t(64)=$ $1.75, P=0.08)$. For the total change from baseline to follow-up there was a non-significant difference of $0.36(s e=0.51)$ with an effect size $(d=0.22)$ in favor of ABFT $(t(64)=-0.72, P=$ $0.48)$.

For clinically meaningful change in depressive symptoms (i.e., BDI-II < 9), we found marginally significant effects and large effect sizes represented at all time points. At midtreatment, $34.4 \%$ of ABFT participants and $11.1 \%$ of EUC participants reported depression scores in the non-clinical range $\left(\mathrm{OR}=4.19 ; 95 \% \mathrm{CI}: 1.03-17.07 ; \chi^{2}(1)=4.38 P=0.04\right)$. At posttreatment, $54.8 \%$ of ABFT participants and $31.0 \%$ of EUC participants reported non-clinical depression scores $\left(\mathrm{OR}=2.70,95 \% \mathrm{CI}\right.$ : 0.94-7.71; $\left.\chi^{2}(1)=3.46, P=0.06\right)$. At follow-up, 58.1\% of ABFT participants and $38.5 \%$ of EUC participants reported non-clinical depression scores $\left(\mathrm{OR}=2.21,95 \%\right.$ CI: $\left.0.76-6.42 ; \chi^{2}(1)=2.17, P=0.14\right)$.

\section{Treatment Retention}

ABFT patients attended a significantly greater number of sessions $(M=9.71, \mathrm{SD}=5.26)$ than EUC patients $(M=2.87, \mathrm{SD}=3.3 ; Z=-4.74, P<.001)$. To determine if treatment rather than the number of sessions impacted the SIQ-JR scores, we explored the trajectory of change for the subsample of patients who received no more than six sessions in either treatment (ABFT $=9 ;$ EUC $=27)$. Similar to the findings in the full sample, analysis of the subsample 
Family Therapy for Suicidal Youth

demonstrated that ABFT cases showed a marginally greater rate of change during the active treatment period compared to $\operatorname{EUC}(F(1,34)=2.84, P=0.10)$.

\section{Subsample of Adolescents Diagnosed with Depression}

To facilitate comparison with other treatment studies, we looked at rates of change on SIQ-JR for those patients who met criteria for clinical depression during the past year $(n=35$, $\left.53.0 \% ; \mathrm{EUC}=19,61.3 \% ; \mathrm{ABFT}=16 ; 45.7 \% ; \chi^{2}(1)=1.60, P=0.21\right)$. Similar to findings from the full data set, ABFT demonstrated a significantly greater rate of change than EUC for these depressed participants during the treatment phase $(F(1,33)=6.16, P=0.02)$. During the followup phase, there was no significant difference in rate of change between the two groups $(F(1,33)$ $=0.10, P=0.75)$. The total change, from baseline to follow-up was $-4.35(s e=0.66)$ for ABFT and -2.19 $(s e=0.62)$ for EUC on the square-root SIQ-JR. This corresponded to a significant difference of $2.16(s e=0.91)$ with an effect size $(d=1.00)$ in favor of $\operatorname{ABFT}(t(33)=-2.39, P=$ 0.02). Therefore, the large effect on suicidal ideation in favor of ABFT for clinically depressed subjects is similar to the effect found for the full sample.

\section{Discussion}

The findings from this study are promising. Compared to usual care in the community, youth treated with ABFT demonstrated significantly greater and more rapid reductions in suicidal ideation during the treatment. These differences persisted at follow-up with an overall large effect size. These findings are strengthened by the consistency across self-report and clinician ratings, and that findings were similar even if patients met criteria for a depressive diagnosis. ABFT was also associated with greater rates of clinical recovery. For this, we used the stringent criteria of scores similar to a non-clinical sample, below 13, rather than the SIQ-JR clinical cut off of 31 . On both the SIQ-JR and the SSI, patients in ABFT at post-treatment and 
Family Therapy for Suicidal Youth

follow-up, were at least 4 times more likely to show scores in recovery than patients in EUC.

This is one of the few studies to demonstrate that a research treatment was more effective than treatment as usual for reducing suicidal ideation in adolescents. ${ }^{3}$

ABFT also demonstrated nearly significant reductions in depressive symptoms by midtreatment compared to EUC, with no difference at post-treatment and follow-up. Effect sizes, however, at all time points were very strong for ABFT, suggesting that a larger sample size may have produced statistical significance. In addition, long term differences between treatments for depression are uncommon, ${ }^{8,14}$ possibly due to the episodic nature of the disorder. Still, ABFT provided more rapid relief from depressive symptoms than community care, an important advantage when youth are at risk for suicide. These findings were supported by the measures of clinical recovery for depression. Compared to EUC, patients in ABFT were 3 times more likely to report BDI-II total scores in the non-clinical range at mid-treatment and 2.3 times more likely at follow-up.

The data on retention was particularly important. ABFT retained adolescents in treatment longer than usual care, even with the additional support and monitoring offered in EUC. These findings are meaningful given the difficulties of engaging suicidal youth in outpatient treatment. While better retention in research based therapy than in community therapy is not surprising, our retention rates were as good as or better than most clinical trials with suicidal, low income, minority youth. ${ }^{3,7,21,22}$ This may be a factor of the highly structured parent engagement strategies of ABFT, but more research is needed to test that hypothesis.

Unfortunately, the low EUC retention rate also raises questions about the internal validity of the study. In other words, what is really causing the change? Differential outcomes may be a function of the treatment dose and not treatment type? Perhaps an equal dose of community care 
would have achieved a similar outcome as ABFT. Alternatively, maybe outcome and retention effects were due to therapist differences. This study compared trained, research therapists receiving close supervision (ABFT) to a range of providers, with varied training backgrounds, working in a range of treatment settings. These two confounds (i.e., dose and therapist differences) plague most studies using TAU as a comparison group. ${ }^{32}$

Despite these limitations, many reasons support enthusiasm for the validity of the results. First, when comparing all patients that received a low dose of either treatment, patients in ABFT continued to show better outcomes. Second, if we compare post-treatment scores on the SIQ-JR across studies, where most samples start above a score of 31 , ABFT $(M=8)$ consistently does as well or better than other experimental treatments: 26 for Peer Nominated Support, ${ }^{5} 24.5$ for skillbased treatment, ${ }^{7} 11.8$ for CBT plus fluoxetine, 14.4 for fluoxetine alone, 11.4 for CBT alone and 15 for placebo. ${ }^{8}$ While these points are encouraging, conclusive claims about the efficacy of ABFT for the treatment of suicidal youth will depend on a future study that can address the design issues outlined above.

Other limitations should be considered. First, assessors were not blind to treatment assignment. Although not uncommon in studies of high-risk participants, ${ }^{29,33}$ the consistency in findings across self-report and interviewer measures, and across multiple clinical domains, lends some confidence that assessor bias did not impact the results. Second, three-fourths of the sample was African American, and nearly half lived below the poverty level. Thus, our findings may not generalize to more affluent or culturally heterogeneous samples. Therefore, research on ABFT with more diverse populations is needed. Third, there is not data at this point to indicate when this treatment might be contraindicted (e.g., family conflict is too high or absent). Certainly, no treatment is perfect for all patients, though attempting to help any family through a suicidal crisis 
seems clinically appropriate. Fourth, the relatively short follow-up (6 months postrandomization) limits our understanding of long-term treatment benefits. Longer follow-up would better reflect treatment benefits. Finally, just to be clear, based on this study, we cannot claim that ABFT prevents suicidal behavior, only that it reduces suicidal ideation.

An important goal of the National Strategy for Suicide Prevention is the development, testing, and dissemination of effective treatments for youth suicide. ${ }^{2}$ Family based interventions have shown efficacy in treating these youth, and ABFT in particular seems a promising treatment for reducing suicidal ideation and depressive symptoms. However, more empirical evidence on the efficacy and mechanisms of change for ABFT is warranted before conclusive claims can be made. 


\section{REFERENCES}

1. Centers for Disease Control and Prevention. Web-based Injury Statistics Query and Reporting System (WISQARS). Available at: http://www.cdc.gov/ncipc/wisqars/default.htm. Accessed October 2008.

2. Institute of Medicine. Reducing Suicide: A National Imperative. Washington, DC: National Academy Press; 2002.

3. Spirito A, Esposito-Smythers C. Attempted and completed suicide in adolescence. Annu Rev Clin Psychol. 2006;2:237-266.

4. Katz LY, Cox BJ, Gunasekara S, Miller AL. Feasibility of dialectical behavior therapy for suicidal adolescent inpatients. J Am Acad Child Adolesc Psychiatry. 2004;43(3):276282.

5. King CA, Kramer A, Preuss L, Kerr DCR, Weisse L, Venkataraman S. Youth-Nominated Support Team for suicidal adolescents (Version 1): A randomized controlled trial. $J$ Consult Clin Psychol. 2006;74(1):199-206.

6. Rathus JH, Miller AL. Dialectical Behavior Therapy adapted for suicidal adolescents. Suicide Life Threat Behav. 2002;32(2):146-157.

7. Donaldson D, Spirito A, Esposito-Smythers C. Treatment for adolescents following a suicide attempt: results of a pilot trial. J Am Acad Child Adolesc Psychiatry. 2005;44(2):113-120.

8. Treatment for Adolescents with Depression Study (TADS) Team. Fluoxetine, cognitivebehavioral therapy, and their combination for adolescents with depression: Treatment for 
Family Therapy for Suicidal Youth

Adolescents with Depression Study (TADS) randomized controlled trial. JAMA. 2004;292(7):807-820.

9. Brent D, Emslie G, Clarke G, et al. Switching to another SSRI or to venlafaxine with or without cognitive behavioral therapy for adolescents with SSRI-resistant depression: the TORDIA randomized controlled trial. JAMA. 2008;299(8):901-913.

10. Tarrier N, Taylor K, Gooding P. Cognitive-behavioral interventions to reduce suicide behavior: a systematic review and meta-analysis. Behav Modif. 2008;32(1):77-108.

11. Hammad TA, Laughren T, Racoosin J. Suicidality in pediatric patients treated with antidepressant drugs. Arch Gen Psychiatry. 2006;63(3):332-339.

12. Wagner BM, Silverman MAC, Martin CE. Family factors in youth suicidal behaviors. Am Behav Sci. 2003;46(9):1171-1191.

13. Brent DA, Perper JA, Goldstein CE, et al. Risk factors for adolescent suicide: a comparison of adolescent suicide victims with suicidal inpatients. Arch Gen Psychiatry. 1988;45(6):581-588.

14. Brent DA, Holder D, Kolko D, et al. A clinical psychotherapy trial for adolescent depression comparing cognitive, family, and supportive therapy. Arch Gen Psychiatry. 1997;54(9):877-885.

15. Harrington R, Kerfoot M, Dyer E, et al. Randomized trial of a home-based family intervention for children who have deliberately poisoned themselves. J Am Acad Child Adolesc Psychiatry. 1998;37(5):512-518.

16. Huey SJ, Henggeler SW, Rowland MD, et al. Multisystemic therapy effects on attempted suicide by youths presenting psychiatric emergencies. J Am Acad Child Adolesc Psychiatry. 2004;43(2):183-190. 
17. Diamond GS, Reis BF, Diamond GM, Siqueland L, Issacs L. Attachment-based family therapy for depressed adolescents: a treatment development study. J Am Acad Child Adolesc Psychiatry. 2002;41(10):1190-1196.

18. Prinstein, M J, Nock, K.M., Simon C, Aikins JW., Cheak CS, Spirto A. Longitudinal trajectories and predictors of adolescent suicide ideation and attempts following inpatient hospitalization. J Consult Clin Psychol. 2008; 76(1): 92-103.

19. Pearson JL, Stanley B, King CA, Fisher CB. Intervention research with persons at high risk for suicidality: safety and ethical considerations. J Clin Psychiatry. 2001;62(suppl 25):17-26.

20. Joe S, Baser RE, Breeden G, Neighbors HW, Jackson JS. Prevalence of and risk factors for lifetime suicide attempts among blacks in the United States. JAMA. 2006;296(17):2112-2123.

21. Rotheram-Borus MJ, Piacentini J, Van Rossem R, et al. Enhancing treatment adherence with a specialized emergency room program for adolescent suicide attempters. $J A m$ Acad Child Adolesc Psychiatry. 1996;35(5):654-663.

22. Spirito A, Boergers J, Donaldson D, Lewander W. An intervention trial to improve adherence to community treatment of adolescent following a suicide attempt. J Am Acad Child Adolesc 2002; Psychiatry 41(4): 435-442.

23. Reynolds WM. Professional Manual for the Suicidal Ideation Questionnaire. Odessa, Fla: Psychological Assessment Resources Inc; 1988.

24. Beck A, Steer R, Brown G. The Beck Depression Inventory-Second Edition. San Antonio, Tex: Psychological Corp; 1996. 
Family Therapy for Suicidal Youth

25. Diamond GS, Siqueland L \& Diamond GM. Attachment-based family therapy: A program of treatment development research. Clinl Child and Famy Psycholy Rev. 2003; 6(2):107-128.

26. Joiner T, Coyne JC, eds. The interactional nature of depression: advances in interpersonal approaches. Washington, DC: American Psychological Association; 1999.

27. Beck AT, Kovacs M, Weissman A. Assessment of suicidal intention: the Scale for Suicide Ideation. J Consult Clin Psychol. 1979;47(2):343-52.

28. Reynolds WM, Mazza JJ. Assessment of suicidal ideation in inner-city children and young adolescents: reliability and validity of the Suicidal Ideation Questionnaire-JR. School Psych Rev.

29. Brown GK, Have TT, Henriques GR, Xie SX, Hollander JE, Beck AT. Cognitive therapy for the prevention of suicide attempts: A randomized controlled trial. JAMA. 2005;294(5):563-570.

30. Wasserman GA, McReynolds LS, Ko SJ, et al. Screening for Emergent Risk and Service Needs Among Incarcerated Youth: Comparing MAYSI-2 and Voice DISC-IV. J Am Acad Child Adolesc Psychiatry. 2004;43(5):629-639.

31. Raudenbush SW, Bryk AS. Hierarchical Linear Models: Applications and Data Analysis Methods. $2^{\text {nd }}$ ed. London: Sage; 2002.

32. Spirito A, Stanton C, Donaldson D, Boergers J. Treatment-as-usual for adolescent suicide attempters: Implications for the choice of comparison groups in psychotherapy research. J Clin Child Adolesc Psychol. 2002; 31(1):41-47. 
Family Therapy for Suicidal Youth

33. Slee N, Garnefski N, van der Leeden R, Arensman E, Spinhoven P. Cognitive-behavioral intervention for self-harm: Randomized controlled trial. Br J Psychiatry. 2008;192:202211. 
Table 1. Patient Characteristics

\begin{tabular}{|c|c|c|c|}
\hline & $\begin{array}{l}\text { EUC } \\
(n=31)\end{array}$ & $\begin{array}{l}\text { ABFT } \\
(n=35)\end{array}$ & $P$ value \\
\hline \multicolumn{4}{|l|}{ Demographic Characteristics } \\
\hline Age, mean (SD), y & $15.29(1.83)$ & $15.11(1.41)$ & .66 \\
\hline AA race, No. $(\%)$ & $24(77.4)$ & $25(71.4)$ & .78 \\
\hline Female sex, No. $(\%)$ & $23(74.2)$ & $32(91.4)$ & .10 \\
\hline Income, less than $\$ 30,000$ yearly income ${ }^{a}$, No. $(\%)$ & $13(44.8)$ & $14(43.8)$ & 1.00 \\
\hline Parent is high-school graduate, No. (\%) & $21(84.0)$ & $26(78.8)$ & .74 \\
\hline Single-parent household, No. (\%) & $22(73.33)$ & $25(73.53)$ & .98 \\
\hline Social Services involvement, No. (\%) & $13(43.3)$ & $12(34.3)$ & .61 \\
\hline
\end{tabular}

\section{Current Psychiatric Diagnoses, No. (\%)}

Major depressive episode

Dysthymia

Any anxiety

Externalizing disorder (ADHD, ODD, CD)

$\begin{array}{lll}13(41.9) & 13(37.1) & .80 \\ 2(6.5) & 3(8.6) & 1.00 \\ 23(74.2) & 21(60.0) & .30 \\ 15(48 \%) & 23(65 \%) & .16\end{array}$

\section{Clinical History, No. (\%)}

Adolescent attempted suicide in the past

$\begin{array}{lll}19(61.3) & 22(62.9) & 1.00 \\ 12(63.2) & 18(81.8) & .29 \\ 7(24.1) & 7(20.0) & .77 \\ 3(10.3) & 3(8.6) & 1.00 \\ 10(34.3) & 10(30.3) & .79\end{array}$

Family history of suicide attempt

$10(34.3)$

\footnotetext{
a - Income: For the entire sample $27(44.3 \%)$ had income under $\$ 30,000 ; 25(41 \%)$ had income $\$ 30,000$ - $\$ 70,000$.

c - Of those with history of attempt

d - None were deaths of parents
} 
Table 2. Rates of Change Estimates

\begin{tabular}{lllll}
\hline & \multicolumn{2}{c}{ PHASE 1 } & \multicolumn{2}{c}{ PHASE 2 } \\
\hline MEASURE & ABFT & EUC & ABFT & EUC \\
& estimate (se) & estimate (se) & estimate (se) & estimate (se) \\
\hline SIQ-JR $^{\text {a }}$ & $-1.77(0.13)$ & $-1.12(0.13)$ & $0.16(0.06)$ & $0.17(0.07)$ \\
SSI $^{\text {a }}$ & $0.84(0.17)$ & $0.26(0.16)$ & $0.12(0.09)$ & $0.06(0.08)$ \\
BDI $^{{ }^{b}}$ & $-1.39(0.18)$ & $-0.94(0.19)$ & $-0.05(0.05)$ & $0.09(0.05)$ \\
SIQ-JR $^{\mathbf{c}}$ & $-1.72(0.21)$ & $-1.08(0.19)$ & $0.14(0.09)$ & $0.17(0.09)$ \\
\hline
\end{tabular}

\footnotetext{
${ }^{\mathrm{a}}$ - PHASE 1 = change during active treatment; PHASE 2 = change during follow-up.

${ }^{\mathrm{b}}$ - PHASE 1 = change during the first six weeks of treatment; PHASE 2 = change from 6 weeks to follow-up. ${ }^{\mathrm{c}}$ - Only depressed participants. PHASES defined as in ${ }^{\mathrm{a}}$.
} 
Table 3. Clinical Response by Treatment

\begin{tabular}{|c|c|c|c|c|}
\hline \multicolumn{5}{|c|}{ Clinical Response Rate \% (CI) } \\
\hline SIQ-JR (<13) & ABFT $(\mathbf{n}=35)$ & EUC $(n=31)$ & Odds Ratio & $P$ value \\
\hline 6 week & $69.7=23 / 33(53.1-86.2)$ & $40.7=11 / 27(20.9-60.5)$ & 3.35 & 0.02 \\
\hline 12 week & $87.1=27 / 31(74.6-99.6)$ & $51.7=15 / 29(32.4-71.1)$ & 6.30 & 0.003 \\
\hline 24 week & $70.0=21 / 30(52.6-87.4)$ & $34.6=9 / 26(15.0-54.2)$ & 4.41 & 0.008 \\
\hline \multicolumn{5}{|l|}{ SSI (0 vs. 1) } \\
\hline 6 week & $40.7=11 / 27(20.9-60.5)$ & $33.3=8 / 24(13.0-53.7)$ & 1.38 & 0.59 \\
\hline 12 week & $69.2=18 / 26(50.2-88.2)$ & $34.6=9 / 26(15.0-54.2)$ & 4.25 & 0.013 \\
\hline 24 week & $82.1=23 / 28(67.0-97.3)$ & $46.2=12 / 26(25.6-66.7)$ & 5.37 & 0.006 \\
\hline \multicolumn{5}{|l|}{ BDI-II ( $\leq 9)$} \\
\hline 6 week & $34.4=11 / 32(17.0-51.8)$ & $11.1=3 / 27(1.6-23.8)$ & 4.19 & 0.04 \\
\hline 12 week & $54.8=17 / 31(36.3-73.4)$ & $31.0=9 / 29(13.1-48.9)$ & 2.70 & 0.06 \\
\hline 24 week & $58.1=18 / 31(39.7-76.8)$ & $38.5=10 / 26(18.4-58.5)$ & 2.21 & 0.14 \\
\hline
\end{tabular}


Family Therapy for Suicidal Youth

Table 4. Means and Confidence Intervals

\begin{tabular}{|c|c|c|c|}
\hline Measure & Assessment & ABFT (n=35) & EUC $(n=31)$ \\
\hline \multirow[t]{4}{*}{ SIQ } & Baseline & $52.1(47.5-56.7)$ & $49.9(44.9-54.9)$ \\
\hline & 6 week & $15.0(7.5-22.5)$ & $22.2(14.9-29.5)$ \\
\hline & 12 week & $5.2(1.6-8.8)$ & $16.2(10.1-22.2)$ \\
\hline & 24 week & $10.4(5.6-15.2)$ & $23.0(15.6-30.4)$ \\
\hline \multirow[t]{4}{*}{ SSI } & Baseline & $14.2(2.1-26.5)$ & $19.4(4.6-34.1)$ \\
\hline & 6 week & $40.7(20.9-60.5)$ & $33.3(13.0-53.7)$ \\
\hline & 12 week & $69.2(50.2-88.2)$ & $34.6(15.0-54.2)$ \\
\hline & 24 week & $82.1(67.0-97.3)$ & $46.2(25.6-66.7)$ \\
\hline \multirow[t]{4}{*}{ BDI } & Baseline & $33.0(29.8-36.2)$ & $33.0(29.7-36.2)$ \\
\hline & 6 week & $16.6(11.5-21.8)$ & $24.5(18.9-30.1)$ \\
\hline & 12 week & $12.6(8.0-17.2)$ & $18.5(12.9-24.0)$ \\
\hline & 24 week & $12.4(7.8-16.9)$ & $16.2(10.4-21.9)$ \\
\hline
\end{tabular}


Family Therapy for Suicidal Youth

Figure 1. Study Participants from Initial Screening Through Analysis

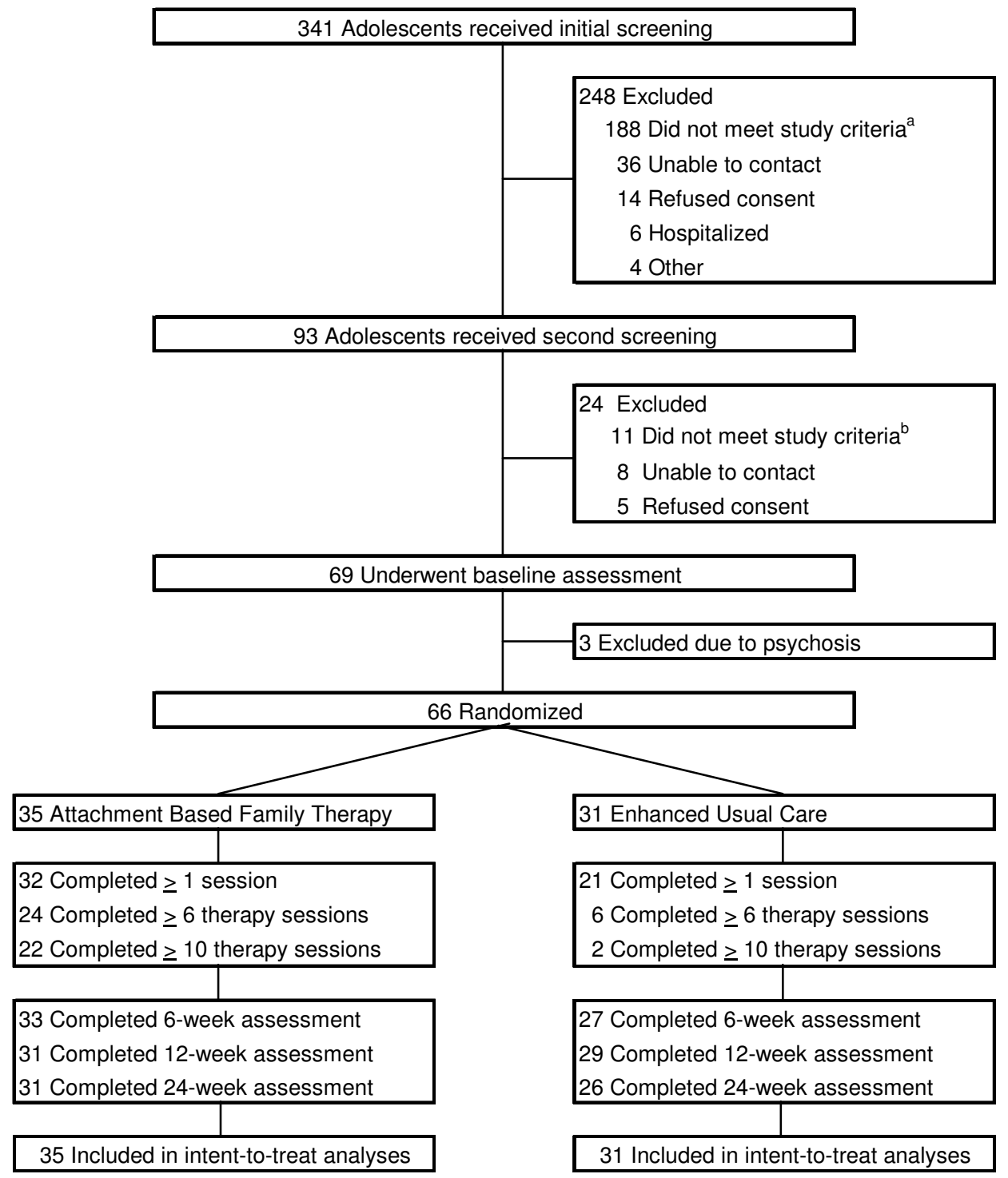


Figure 2: SIQ-JR. profile over time (square-root transformed)

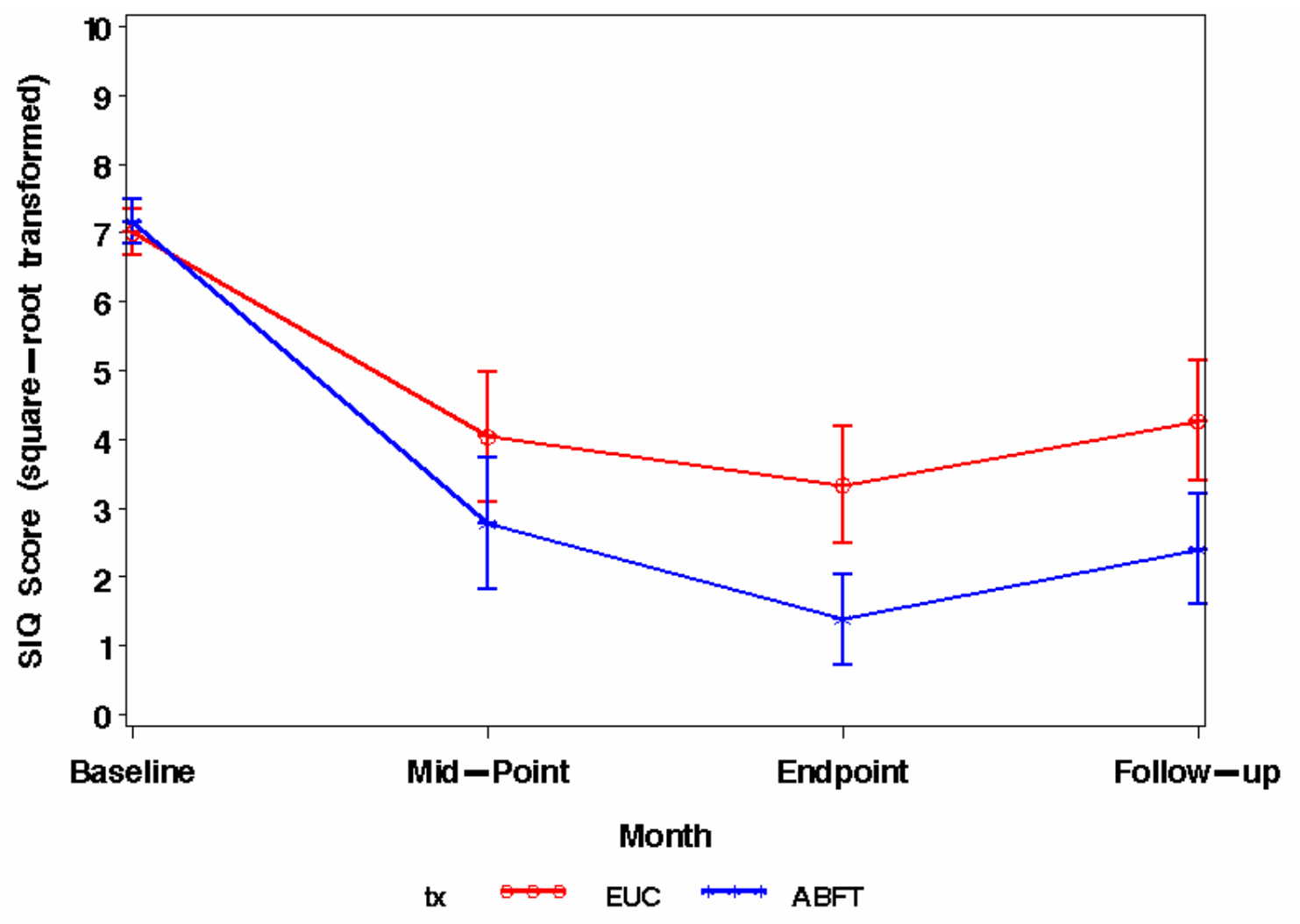

